\title{
Ein grundlegendes Werk zur Deklination im Udmurtischen
}

Н. В. Кондратьева: Категория падежа имени существительного в удмуртском языке. [Die Kasuskategorie des Substantivs im Udmurtischen.] Ижевск: Удмуртский государственный университет, 2011. $255 \mathrm{~S}$.

Natalia Kondrat'eva ist die jüngste Vertreterin der finnisch-ugrischen Völker, die (mit 35 Jahren) in Russland im Bereich der nationalen Wissenschaften promoviert hat. Das hier zu besprechende Werk ist eine der drei Monografien, aufgrund derer ihr der Doktortitel zuerkannt wurde. Kondrat'eva will sich in diesem Buch vor allem aus der Perspektive der traditionellen Grammatikauffassung mit grundlegenden Fragen befassen, weil selbst die Darstellung der Grundstruktur des Udmurtischen nach Festigung verlangt.

Bei einer thematisch so ausgreifenden Abhandlung ist die Eingrenzung besonders wichtig. Man muss abwägen, was einbezogen wird und wie tief die Untersuchung vordringen kann. Das Werk sollte ausgewo- gen sein, was in diesem speziellen Fall u. a. bedeutet, dass die 15 Kasus der udmurtischen Schriftsprache in gleichem Maß behandelt werden. Die Abfassung thematisch breit gefasster Werke ist eine undankbare Aufgabe, weil Spezialisten einzelner Bereiche wohl immer Detailfehler oder Lücken bemängeln werden. Kondrat'eva ist es gelungen, eine anschauliche Darstellung des kompakten udmurtischen Kasussystems vorzulegen, in der auch die Verwendungskontexte einzelner Kasus ausgesprochen genau erläutert werden. Erwähnung finden auch die wichtigsten Dialektunterschiede, die in der Anzahl der Kasus, ihrer Verwendung und der Form der Kasusendungen festzustellen sind. Auch die Tendenzen der historischen Entwicklung der Kasus werden berücksichtigt, sofern sie für das Verständnis der heutigen Situation entscheidend sind.

Die Grundstruktur des Werks ist klar und übersichtlich. Nach der Einleitung, einem allgemeinen sprachwissenschaftlichen Überblick und einem Gesamtüberblick über 
das udmurtische Kasussystem werden die acht grammatischen Kasus und die sieben Lokalkasus in separaten Unterkapiteln behandelt. Jeder Kasus wird zuerst aus morphologischer Sicht behandelt, dann werden die Dialektunterschiede kurz erwähnt und anschließend wird die Funktion des Kasus betrachtet. In eigenen Kapiteln werden auch die sekundäre Flexion und der Komparativ-Moderativ -zec behandelt. Im letzten Kapitel werden die wichtigsten Punkte noch einmal zusammenfassend dargestellt.

\section{Traditionelle Untersuchung der Sprachstruktur}

Die Abhandlung hat das Ziel, den derzeitigen Stand der Forschung über die Kasus des Udmurtischen zusammenzufassen und auf der Basis dieser Kenntnisse das System ganzheitlich zu behandeln. Damit leistet Kondrat'eva der wissenschaftlichen Gemeinschaft einen großen Dienst. Man darf annehmen, dass künftig oft auf ihr Werk verwiesen wird. Als theoretischer Hintergrund werden neben Untersuchungen über das Udmurtische auch grammatische Werke über das Russische und Abhandlungen über türkische Sprachen sowie allgemeinlinguistische Werke verwendet. Diese werden sinnvoll genutzt. Es finden sich kaum störende Lapsus, allerdings einige Druckfehler. Die Zusammenfassung auf den Seiten 208-209 hätte in einem eigenen Kapitel präsentiert werden sollen. Jetzt findet sie sich in einem Kapitel, das der Überschrift zufolge nur die temporalen Bedeutungen des Approximativs behandeln sollte. Alternativ hätte man diese Schlussfolgerungen auch dem letzten Kapitel (S. 232-234) anfügen können. Die Publikation von Kuznecova (2005), auf die S. 146 verwiesen wird, fehlt im Literaturverzeichnis.

Die Arbeit präsentiert nicht nur die bisherige Forschung über das Udmurtische, sondern zeigt auch, wie spärlich und lückenhaft viele Elemente des Udmurtischen im Vergleich etwa zum Russischen oder Finnischen erforscht sind. Dies konstatiert Kondrat'eva an einigen Stellen auch ausdrücklich; z. B. gibt es keine Untersuchungen über das im Genitiv stehende Agens im Udmurtischen (S. 80). Viele Kasusformen können im Udmurtischen durch Postpositionskonstruktion ersetzt werden (S. 105, 157, 170, 201, 204). Diese Variation ist offensichtlich kaum untersucht worden. Kondrat'eva kann nur auf vereinzelte Beobachtungen verweisen, wonach in einigen Dialekten anstelle mancher Kasus Postpositionen auftreten; so verwendet man z. B. in den peripheren Süddialekten anstelle des Approximativs die Postposition пала (S. 146). Des weiteren 
treten mehrere andere Variationserscheinungen hervor, bei denen die Voraussetzungen für die Verwendung unbekannt sind: z. B. können bei einigen auf - $a$ auslautenden Wörtern die Lokalkasus endungslos sein oder eine Endung haben (куала / куалае 'an den Haus') (S. 154, 163), der Prolativ kann mit der Endung -emu oder - $m u$ gebildet werden (капкаети / капкати 'aus dem Tor') (S. 195) usw. Es hat den Anschein, dass die Variationsforschung bisher gar nicht auf der Agenda der Udmurtologie gestanden hat, außer in Fällen, wo die Variation auf Dialektunterschieden beruht oder ein konkreter lexikalischer oder phonetischer Grund erkennbar ist.

Eine sehr interessante Variation begegnet in Sätzen, die zum Ausdruck bringen, dass etwas aus etwas gemacht wird. Im Udmurtischen ist in solchen Sätzen ein zweifaches Objekt möglich: Der mit Kasusendung markierte Akkusativ bezeichnet das Ausgangsmaterial, der endungslose Akkusativ das Ergebnis der Handlung. Anstelle des erstgenannten kann auch der Elativ oder der Ablativ verwendet werden (S. 52-53). Kondrat'eva behandelt diese Fälle, als ginge es um synonyme Ausdrükke und als sei die Variation nur eine Art vorübergehende Erscheinung, die durch das Streben der Kasus nach semantischer Klarheit allmählich verschwindet. Es wäre frucht- barer, sich diesem Phänomen aus der Perspektive anzunähern, dass ein Sachverhalt dieser Art (infolge einer Handlung A > B) nach unterschiedlichen Schemata versprachlicht werden kann: aus Eisen eine Axt schmieden / Eisen zu einer Axt schmieden. Zudem kann es für die Variation von Akkusativ, Elativ und Ablativ Gründe geben, die z. B. mit der Informationsstruktur des Satzes in Verbindung stehen. Ein zweiter, ebenso interessanter Fall ist der Wechsel von Instrumental, Adverbial und Nominativ (S. 143).

In separaten Kapiteln betrachtet Kondrat'eva auch die sekundäre Flexion im Udmurtischen und die Verwendung des Suffixes -zec bei Substantiven. Unter sekundärer Flexion ist $\mathrm{zu}$ verstehen, dass ein Wort zwei (oder mehr) Kasusendungen hat. Dieses u. a. auch im Komi und in den mordwinischen Sprachen mögliche Phänomen erklärt sich daraus, dass in diesen Fällen das mit Kasusendung versehene Wort als Wortstamm fungieren kann, so wie der eigentliche Wortstamm. Die erste Kasusendung hat also Wortbildungsfunktion. In diesen Fällen tritt häufig auch ein Px. oder Determinativsuffix an das Wort, doch dies ist nicht obligatorisch. Im Udmurtischen ist diese Erscheinung vor allem mit der Ellipse verknüpft (S. 210-223). Die übersichtliche Darstellung dieses Phänomens zählt zu 
den besten Partien des vorliegenden Werks. Dasselbe gilt für das Kapitel über die traditionell als Komparativ bezeichnete Endung -2ec. Neben der komparativen hat sie auch die moderative Bedeutung 'fast, beinahe, drückt dann also bei den Adjektiven nicht eine größere Quantität der betreffenden Eigenschaft aus, sondern im Gegenteil eine geringere. Bei den Substantiven hat der Moderativ eine ähnliche Bedeutung, z. В. гармошка-ен-гес шудыны '(im Allgemeinen, gewöhnlich) Ziehharmonika spielen'. Kondrat'eva vertritt die Auffassung, dass es sich in diesen Fällen nicht mehr um einen Vergleich handelt. Wie wäre dann aber zu erklären, dass dasselbe Suffix in zweierlei Bedeutung verwendet wird? Meiner Ansicht nach handelt es sich in beiden Fällen um einen Vergleich, nur ist (natürlich in der Tiefenstruktur) der Vergleichspunkt ein anderer: Beim Komparativ ist er $+\mathrm{X}$ (z. B. größer als etwas Großes), beim Moderativ dagegen -X (z. B. größer als nicht-groß, d. h. eher groß als klein, ziemlich groß). Ebenso verhält es sich bei den Substantiven: гармошка-ен-гес шудыны bеdeutet eher Ziehharmonika spielen als nicht spielen.

\section{Die Bezeichnungen der Kasus}

Kondrat'eva unterzieht die Bezeichnungen für die Kasus des Udmurti- schen einer kritischen Betrachtung. In der russischen Altaistik wurde die Bezeichnung именительный падеж kritisiert, u. a. deshalb, weil der Nominativ in den altaischen Sprachen eine weiterreichende Funktion hat als im Russischen; als Ersatz wurden andere Termini vorgeschlagen. Kondrat'eva erkennt die Kritik als berechtigt an, hält jedoch an den traditionellen Bezeichnungen fest (S. 26-27). Für den Genitiv bevorzugt sie jedoch statt именительный падеж die Bezeichnung притяжательный падеж, weil er in der Regel den Besitzer markiert. Aus demselben Grund empfiehlt sie auch, den udmurtischen Terminus zu ändern. Sie selbst verwendet jedoch noch die traditionellen Bezeichnungen, weil ihre Vorschläge vorläufig nur Empfehlungen sind (S. 66). Auch über die Bezeichnung für den Ablativ wurden unter Udmurtologen rege Debatten geführt und zahlreiche Termini vorgeschlagen (S. 83). Kondrat'evas Standpunkt erscheint gerechtfertigt. Termini müssen kritisch betrachtet werden, und es ist gut, ihren Hintergrund zu kennen; dies kann auch zum Verständnis des jeweiligen Phänomens an sich beitragen. In der Regel ist es nicht sinnvoll, von einem etablierten Terminus abzurücken, doch wenn gewichtige Gründe vorliegen, kann man es tun, wie bei den russischen und udmurtischen Bezeichnun- 
gen für den udmurtischen Genitiv. Ein gutes Beispiel für eine bereits erfolgte Veränderung eines Terminus bietet gerade der Genitiv. Die frühen Forscher, insbesondere die Finnen, bezeichneten ihn als Adessiv, weil er vom Ursprung her dem finnischen Adessiv entspricht und zum Teil in den gleichen Kontexten (S. 66) verwendet wird, z. B. in Besitzkonstruktionen. Diese Lösung ist durchaus verständlich, denn in der Forschung stand damals der historisch-vergleichende Aspekt im Vordergrund. Als die Beschreibung der udmurtischen Sprache in den Fokus trat, wurde die Bezeichnung Adessiv unhaltbar.

\section{In Erwartung einer hand- lichen Grammatik}

Die vorliegende Arbeit fungiert auch als Handbuch der Deklination und ist sicherlich tauglich für Wissenschaftler, die die benötigten Informationen $\mathrm{zu}$ suchen wissen und mit dem Stil der sprachwissenschaftlichen Argumentation vertraut sind. Unabhängig von der Leserschaft hätte man die Lesbarkeit mühelos verbessern können, indem man die Anzahl der Beispiele verringert und sie vom Fließtext getrennt präsentiert hätte. So werden etwa zum Gebrauch des Dativs (S. 116) und des Abessivs (S. 135) acht Beispiele gegeben, die einander ausgesprochen ähnlich sind und nicht im Einzelnen kommentiert werden. An vielen Stellen wäre es angebracht gewesen, die Angaben in Form einer Tabelle oder eines Diagramms zu komprimieren. Im vorliegenden Buch fehlt sogar eine Tabelle, in der alle Kasusformen zusammengefasst sind.

In letzter Zeit sind mehrere Lehrbücher des Udmurtischen veröffentlicht worden. Es gibt Lehrwerke für Schüler, sowohl für den muttersprachlichen Unterricht als auch für Udmurtisch als Zweitsprache. Auch speziell für ausländische an der Sprache Interessierte liegen bereits mehrere Lehrbücher und Sprachbeschreibungen vor. Die Terminologiekommission hat Neologismenverzeichnisse veröffentlicht, und Untersuchungen über das Udmurtische erscheinen in stetiger Folge. Handliche, für jeden am Udmurtischen Interessierten konzipierte Handbücher, Sprachführer oder zeitgemäße Grammatiken sind in Udmurtien nicht erhältlich. Kondrat'evas Werk würde sich als Material für eine russisch- und udmurtischsprachige Gebrauchsgrammatik des Udmurtischen eignen. Dazu müssten natürlich die für das breite Publikum überflüssigen Hintergrundinformationen gestrichen und die sprachwissenschaftliche Argumentation vereinfacht werden. Außerdem müsste man 
ein Verzeichnis der verwendeten den visuellen Eindruck und die Termini samt Erklärungen und Benutzerfreundlichkeit verbessern. Verweisen auf die entsprechenden Ein solches Buch ist ein unbedingStellen der Grammatik hinzufügen tes Desiderat. und neben Tabellen und Diagrammen weitere Lösungen finden, die 